\title{
Trabalho de parto: usos de métodos não farmacológicos para alívio da dor
}

\author{
Labor: uses of non-pharmacological methods for pain relief
}

Alumbramiento: usos de métodos no farmacológicos para aliviar el dolor

Larissa Toloy Bigaran

ORCID: https://orcid.org/0000-0001-5815-4000

Universidade Brasil, Brasil

E-mail: larissatoloyb@gmail.com

Larissa Barros de Almeida

ORCID: https://orcid.org/0000-0003-2364-9892

União das Faculdades dos Grandes Lagos, Brasil

E-mail: larissabarrosfai@gmail.com

Talita Costa Barbosa

ORCID: https://orcid.org/0000-0002-0396-0651

Universidade Brasil, Brasil

E-mail: talitacostabarbosa@gmail.com

Izadora Amorim de Souza

ORCID: https://orcid.org/0000-0002-3048-4267

União das Faculdades dos Grandes Lagos, Brasil

E-mail: izadoraamorim.souza@gmail.com

Andressa Andrade de Souza

ORCID: https://orcid.org/0000-0002-8234-2789

União das Faculdades dos Grandes Lagos, Brasil

E-mail: andressaandrade100@ hotmail.com

Felipe Custódio Teixeira

ORCID: https://orcid.org/0000-0002-8847-3173 Universidade Brasil, Brasil

E-mail: felipe_teixeiract2@hotmail.com

Marcos Flávio Silva Filho

ORCID: https://orcid.org/0000-0003-0772-109X Universidade Brasil, Brasil

E-mail: tatosilva10@yahoo.com.br

Letícia Marin Mendes

ORCID: https://orcid.org/0000-0002-5964-1879 Universidade Brasil, Brasil

E-mail: le.ty.leticia16@gmail.com

Thais Cardoso Menis

ORCID: https://orcid.org/0000-0002-7162-2320 Universidade Brasil, Brasil

E-mail: menisthais@gmail.com

Letícia Okasaki Reis

ORCID: https://orcid.org/0000-0002-1680-4471 Universidade Brasil, Brasil

E-mail: leticiaokazaki20@gmail.com

Giovana Maschietto Sussai

ORCID: https://orcid.org/0000-0001-9604-4419 Universidade Brasil, Brasil

E-mail: giovanamaschiettosussai@gmail.com

Mylena Aparecida Silva de Camargo

ORCID: https://orcid.org/0000-0002-6351-2420 Universidade Brasil, Brasil

E-mail: camargomy2109@gmail.com

Gabriel Henrique Cardoso Silva

ORCID: https://orcid.org/0000-0002-3614-0385

Faculdade de Medicina de Catanduva, Brasil

E-mail: gabriel_henriquec@ hotmail.com

Fausto da Silva Gonçalves

ORCID: https://orcid.org/0000-0001-5188-0536

União das Faculdades dos Grandes Lagos, Brasil

E-mail: faustosgoncalves@gmail.com

Elizabete Santos Melo

ORCID: https://orcid.org/0000-0002-7322-9370

Universidade Brasil, Brasil

E-mail: enfaelizabetemelo@gmail.com 


\begin{abstract}
Resumo
O parto representa o último estágio da concepção no organismo materno. Durante esse período, a parturiente vivencia uma das percepções dolorosas mais marcantes, cabendo aos profissionais, de forma humanizada, trazer alívio à mulher. A dor é uma experiência emocional e sensorial desagradável, associada a lesões reais ou potenciais. Sob esse aspecto, busca-se suprir a assistência humanizada, que tem extrema importância, com o uso de métodos para alivio da dor, que não levarão a gestante a passar por uma gama de intervenções farmacológicas, o qual muitas vezes podem ser desnecessárias, visto que é de suma importância quando se pensa na importância de manter o estado físiológico. Diante disso, realizou-se uma revisão integrativa de literatura, descrita como um método de pesquisa que permite a busca, avaliação crítica e a síntese das evidencias disponíveis do tema estudado. Nesse aspecto, é possível perceber que já é conhecido que existem métodos não farmacológicos que favorecem efetivamente na diminuição da dor e da ansiedade durante o parto. Logo, sugere-se mais pesquisas sobre os benefícios de tais métodos, confirmando que estas técnicas são realmente eficazes para a redução da dor e da ansiedade decorrentes do período de parto e possam, dessa forma, serem mais aplicadas nos serviços de assistência obstétrica.
\end{abstract}

Palavras-chave: Métodos não farmacológicos; Dor; Parto.

\begin{abstract}
Childbirth represents the last stage of conception in the maternal organism. During this period, the parturient experiences one of the most striking painful perceptions, and it is up to the professionals, in a humanized way, to bring relief to the woman. Pain is an unpleasant emotional and sensory experience, associated with actual or potential injuries. In this regard, we seek to provide humanized care, which is extremely important, with the use of methods for pain relief, which will not lead the pregnant woman to undergo a range of pharmacological interventions, which may often be unnecessary, as which is of paramount importance when thinking about the importance of maintaining the physiological state. Therefore, an integrative literature review was carried out, described as a research method that allows for the search, critical assessment and synthesis of available evidence on the topic studied. In this aspect, it is possible to see that it is already known that there are non-pharmacological methods that effectively help to reduce pain and anxiety during childbirth. Therefore, further research is suggested on the benefits of such methods, confirming that these techniques are really effective in reducing pain and anxiety resulting from the delivery period and can, therefore, be more applied in obstetric care services.
\end{abstract}

Keywords: Non-pharmacological methods; Pain; Labor.

\title{
Resumen
}

El parto representa la última etapa de la concepción en el organismo materno. Durante este período, la parturienta experimenta una de las percepciones dolorosas más llamativas, y corresponde a los profesionales, de manera humanizada, brindar alivio a la mujer. El dolor es una experiencia emocional y sensorial desagradable, asociada con lesiones reales o potenciales. En este sentido, buscamos brindar una atención humanizada, que es de suma importancia, con el uso de métodos de alivio del dolor, que no llevarán a la gestante a someterse a un abanico de intervenciones farmacológicas, que muchas veces pueden resultar innecesarias, ya que es de de suma importancia a la hora de pensar en la importancia de mantener el estado fisiológico. Por ello, se realizó una revisión integradora de la literatura, descrita como un método de investigación que permite la búsqueda, valoración crítica y síntesis de la evidencia disponible sobre el tema estudiado. En este aspecto, se puede ver que ya se sabe que existen métodos no farmacológicos que ayudan eficazmente a reducir el dolor y la ansiedad durante el parto. Por lo tanto, se sugiere una mayor investigación sobre los beneficios de dichos métodos, confirmando que estas técnicas son realmente efectivas para reducir el dolor y la ansiedad resultantes del período de parto y, por lo tanto, pueden ser más aplicadas en los servicios de atención obstétrica.

Palabras clave: Métodos no farmacológicos; Dolor; Alumbramiento.

\section{Introdução}

O parto representa o último estágio da concepção em que o ser gerado iniciará uma vida independente do organismo materno. Durante esse período de transição entre a vida intra e extrauterina, a parturiente vivencia uma das percepções dolorosas mais marcantes -se não a mais- de sua vida, cabendo aos profissionais que a rodeiam, de forma humanizada, trazer alívio à mulher (Osório et al., 2014).

O comitê de taxonomia da Associação Internacional para o Estudo da Dor (Internacional Association for the Studs of Pain - IASP) define dor como uma "experiência emocional e sensorial desagradável, associada a lesões reais ou potenciais, ou descrita em termos dessas lesões". Este conceito considera que a interpretação e a resposta da dor são influenciadas também pela dimensão emocional e não apenas sensorial (Almeida et al., 2008). 
Desse modo, buscando sempre suprir a assistência humanizada, que tem extrema importância por si só, o uso de métodos para alívio da dor, que não levarão a gestante a passar por uma cascata de intervenções farmacológicas, muitas vezes desnecessárias, é de suma relevância para o sucesso, quando se pensa na importância de manter o estado fisiológico (Da et al., 2015). Quando não há tal percepção da necessidade de evitar intervenções desnecessárias, expõe-se a gestante ao risco de, por exemplo, uma episiotomia (sem evidência científica de seus benefícios), tricotomia e a desumana e violenta manobra de Kristeller.

Os métodos não farmacológicos utilizados para o alivio da dor durante o trabalho de parto, são tecnologias de cuidados que envolvem alguns conhecimentos estruturados. $\mathrm{O}$ uso de tais métodos vem sendo alvo de estudos desde a década de 60, e passam a ser introduzidos em algumas instituições a partir da década de 90, com o movimento de parto humanizado. Esses métodos são classificados como tecnologia leve-dura, uma vez que está baseada nos saberes estruturados, tanto dos profissionais de saúde como em relação à clínica e a epidemiologia, organizando sua atuação no processo de trabalho (Gayeski \& Brüggemann, 2010).

A partir disso, a Organização Mundial da Saúde (OMS) recomendou mudanças nessa assistência demasiadamente intervencionista (Leal et al., 2014) e alguns estudos evidenciaram a existência e a eficácia de formas não farmacológicas que visam aliviar a dor do parto, como banho morno, exercícios respiratórios, massagem lombossacral, bola suíça, aromaterapia e deambulação (Mascarenhas et al., 2019).

Segundo o Ministério da Saúde, a partir da Portaria no 1.459, de 24 de junho de 2011, instituiu-se a Rede Cegonha no âmbito do Sistema Único de Saúde (SUS), na qual são adotadas medidas para promoção de melhorias na assistência prestada às mulheres e crianças. De acordo com a demanda, surgiu-se a necessidade de profissionais especializados na área para fornecer todo o suporte necessário para este público alvo. A prática humanizada na assistência ao parto tem o intuito de implementar um acompanhamento emocional, psicológico e familiar, e utilizar métodos não medicamentosos para a diminuição da dor. Executam-se práticas como massagens na região lombar nas parturientes, exercícios para auxiliar na evolução do trabalho de parto, utilização de bola suíça e, o mais importante, deixar a mulher ser a protagonista do processo, respeitando a sua individualidade e desejos (Oliveira et al., 2020).

Mulheres com gravidez sem complicações podem se beneficiar com a prática de exercícios físicos durante a gravidez. Uma atividade bem rotineira nesse público é a dança aeróbica, que pode ser um exercício de baixo impacto e risco mínimo do qual elas podem participar. Alguns estudos relatam que a dança pode ser vista como algo relaxante, alegre, fortalecedor e que oferece uma conexão entre a mãe e seu bebê em desenvolvimento. Ademais, de acordo com alguns estudos, isso pode reduzir a dor, ajudar na progressão do trabalho de parto e promover a satisfação da paciente (Horter et al., 2020).

A assistência ao parto normal acaba se restringindo a ambientes hospitalares. O parto que é para ser considerado natural, acaba sendo visto como patológico, o que se torna desvantajoso, por se submeter a procedimentos desnecessários. Para melhor eficácia desse momento, faz-se necessário a participação de um profissional qualificado que saiba manejar o método adequado para cada situação, com isso deixam as puérperas mais motivadas. O estabelecimento do bem estar físico faz com que as mesmas aceitem de maneira mais tranquila os métodos não farmacológicos, assegurando um parto mais confortável e rápido, e com isso ter um parto humanizado. (Santos et al., 2021).

\section{Metodologia}

O estudo realizado é uma revisão integrativa da literatura, descrita como um método de pesquisa que permite a busca, a avaliação crítica e a síntese das evidências disponíveis do tema investigado (Mendes et al., 2008). Os descritores utilizados foram "non-pharmacological" and "pain labor". Foram encontrados cento e vinte e dois (122) artigos e, desses, 
selecionados treze (13), nos idiomas inglês e português. Para alcance dos estudos escolhidos, as plataformas usadas foram PubMed e SciELO.

Em relação aos critérios de inclusão e exclusão, utilizou-se como critérios de inclusão: artigos disponíveis com publicação nos idiomas português e ing qu que abordassem o tema do estudo e que foram publicados nos últimos cinco anos. A exclusão baseou-se em artigos os quais citavam as terapias medicamentosas para alívio da dor do trabalho de parto.

\section{Resultados e Discussão}

Através das buscas efetivadas nas bases de dados, encontraram-se 113 artigos na PubMed e 9 artigos na SciELO, totalizando 116 artigos, que após aplicação dos critérios de inclusão e exclusão, reduziram-se à 13 documentos, os quais foram utilizados para a confecção da presente revisão; sendo eles, 10 artigos retirados da base PubMed e 3 da base SciELO, nos idiomas inglês e português.

Para melhor esclarecimento de todo método de busca e escolha dos artigos utilizados, optou-se pelo uso de um fluxograma, ilustrado na Figura 1.

Figura 1. Fluxograma do método de busca e escolha dos artigos.

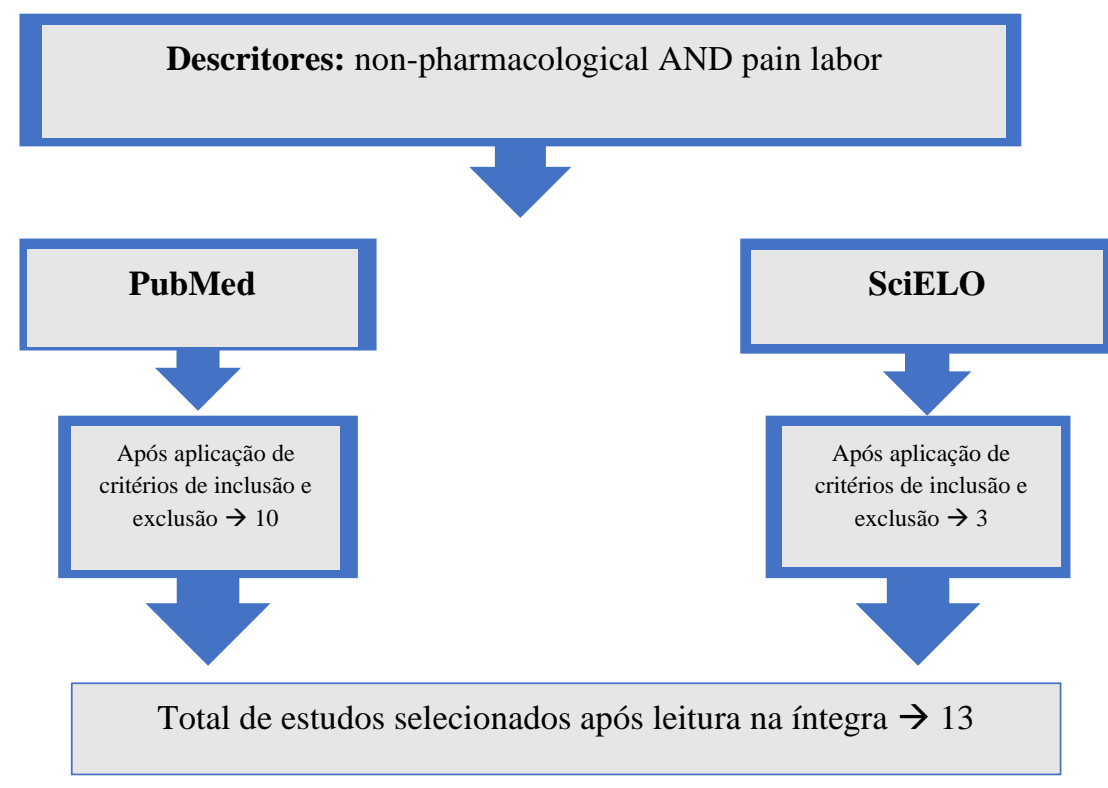

Fonte: Protocolo PRISMA adaptado.

Após leitura dos artigos, optou-se pela inserção dos dados obtidos em uma tabela (Quadro 1), que contém informações como autores e ano, título, objetivo e perfil da amostra, utilizados a fim de organizar e demonstrar os dados colhidos. 
Quadro 1 - Artigos utilizados para a revisão.

\begin{tabular}{|c|c|c|c|}
\hline AUTORES E ANO & TÍTULO & OBJETIVO & PERFIL DA AMOSTRA \\
\hline $\begin{array}{l}\text { Santiváñez-Acosta et } \\
\text { al., } 2020\end{array}$ & $\begin{array}{l}\text { Music Therapy in Pain and } \\
\text { Anxiety Management } \\
\text { during Labor: A Systematic } \\
\text { Review and Meta-Analysis }\end{array}$ & $\begin{array}{l}\text { Mostrar a eficácia da musicoterapia no } \\
\text { controle da dor e da ansiedade durante o } \\
\text { trabalho de parto; o quão eficaz é a } \\
\text { musicoterapia na redução da dor e da } \\
\text { ansiedade, quando comparada ao tratamento } \\
\text { padrão e o quão eficaz é a musicoterapia no } \\
\text { alívio da dor. }\end{array}$ & $\begin{array}{l}\text { É uma revisão sistemática e meta- } \\
\text { análise; incluiu ensaios clínicos } \\
\text { randomizados e / ou estudos quase- } \\
\text { experimentais com informações } \\
\text { completas e acessíveis. As bases de } \\
\text { dados bibliográficas utilizadas foram } \\
\text { PubMed / Medline, LILACS e } \\
\text { COCHRANE, e em buscadores como } \\
\text { TRIPDATABASE e Google Scholar. }\end{array}$ \\
\hline $\begin{array}{l}\text { Almaz Aklilu Getu et } \\
\text { al., } 2020\end{array}$ & $\begin{array}{l}\text { Non-pharmacological labor } \\
\text { pain management and } \\
\text { associated factor among } \\
\text { skilled birth attendants in } \\
\text { Amhara Regional State } \\
\text { health institutions, } \\
\text { Northwest Ethiopia }\end{array}$ & $\begin{array}{l}\text { Determinar a utilização do manejo não } \\
\text { farmacológico da dor do parto e fatores } \\
\text { associados entre parteiras qualificadas em } \\
\text { instituições de saúde do Estado Regional de } \\
\text { Amhara, Etiópia. }\end{array}$ & $\begin{array}{l}\text { Estudo transversal com } 592 \text { parteiras } \\
\text { qualificadas que trabalham na região de } \\
\text { Amhara, Etiópia. }\end{array}$ \\
\hline Smith et al., 2012 & $\begin{array}{l}\text { Massage, reflexology and } \\
\text { other manual methods for } \\
\text { pain management in labor }\end{array}$ & $\begin{array}{l}\text { Examinar os efeitos dos métodos manuais } \\
\text { de cura, incluindo massagem e reflexologia } \\
\text { para o controle da dor durante o trabalho de } \\
\text { parto, na morbidade materna e perinatal. }\end{array}$ & $\begin{array}{l}\text { Revisão de literatura usando o } \\
\text { Cochrane Pregnancy and Childbirth } \\
\text { Group's Trials Register, o Cochrane } \\
\text { Central Register of Controlled Trials } \\
\text { (CENTRAL), a MEDLINE, CINAHL, } \\
\text { o Australian and New Zealand Clinical } \\
\text { Trial Registry, Chinese Clinical Trial } \\
\text { Register, Current Controlled Trials, } \\
\text { ClinicalTrials.gov, ISRCTN Register, } \\
\text { National Center for Complementary } \\
\text { and Alternative Medicine (NCCAM) e } \\
\text { a WHO International Clinical Trials } \\
\text { Registry Platform. }\end{array}$ \\
\hline $\begin{array}{l}\text { Mahbubeh } \\
\text { Tabatabaeichehr \& } \\
\text { Hamed Mortazavi, } \\
2020\end{array}$ & $\begin{array}{l}\text { The Effectiveness of } \\
\text { Aromatherapy in the } \\
\text { Management of Labor Pain } \\
\text { and Anxiety: A Systematic } \\
\text { Review }\end{array}$ & $\begin{array}{l}\text { Revisar sistematicamente as evidências } \\
\text { atualmente disponíveis avaliando o uso da } \\
\text { aromaterapia para o tratamento da dor e } \\
\text { ansiedade do parto. }\end{array}$ & $\begin{array}{l}\text { Revisão sistemática utilizando } 5 \text { bancos } \\
\text { de dados (PubMed, SCOPUS, Web of } \\
\text { Science, Google Scholar e Scientific } \\
\text { Information Database (SID). }\end{array}$ \\
\hline Raana \& Fan, 2020 & $\begin{array}{l}\text { The effect of acupressure } \\
\text { on pain reduction during } \\
\text { first stage of labor: A } \\
\text { systematic review and } \\
\text { meta-analysis }\end{array}$ & $\begin{array}{l}\text { Avaliar criticamente o efeito da acupressão } \\
\text { na redução da dor durante a primeira fase do } \\
\text { trabalho de parto. }\end{array}$ & $\begin{array}{l}\text { Cinco principais bancos de dados } \\
\text { eletrônicos foram pesquisados, } \\
\text { utilizando apenas ensaios clínicos } \\
\text { randomizados comparando acupressão } \\
\text { com placebo e/ou nenhuma } \\
\text { intervenção foram selecionados. }\end{array}$ \\
\hline Smith et al., 2018 & $\begin{array}{l}\text { Relaxation techniques for } \\
\text { pain management in labor }\end{array}$ & $\begin{array}{l}\text { Examinar os efeitos das técnicas de } \\
\text { relaxamento mente-corpo para o controle da } \\
\text { dor durante o trabalho de parto no bem-estar } \\
\text { materno e neonatal durante e após o parto. }\end{array}$ & $\begin{array}{l}\text { Revisão de literatura usando o } \\
\text { Cochrane Pregnancy and Childbirth's } \\
\text { Trials Register, o Cochrane Central } \\
\text { Register of Controlled Trials, } \\
\text { MEDLINE, CINAHL, o Registro de } \\
\text { Ensaios Clínicos da Nova Zelândia da } \\
\text { Austrália, ClinicalTrials.gov, o } \\
\text { Registro ISRCTN e a Plataforma } \\
\text { Internacional de Registro de Ensaios } \\
\text { Clínicos da OMS. }\end{array}$ \\
\hline Smith et al., 2020 & $\begin{array}{l}\text { Acupuncture } r \text { or } \\
\text { acupressure for relieving } \\
\text { pain during labor }\end{array}$ & $\begin{array}{l}\text { Examinar as evidências de ensaios clínicos } \\
\text { randomizados sobre o uso de acupuntura ou } \\
\text { acupressão para ajudar as mulheres a } \\
\text { controlar a dor durante o trabalho de parto. }\end{array}$ & $\begin{array}{l}28 \text { estudos relatando em } 3.960 \\
\text { mulheres comparando a acupuntura } \\
\text { ou acupressão com o tratamento } \\
\text { simulado como placebo, nenhum } \\
\text { tratamento ou cuidado usual para o } \\
\text { controle da dor durante o trabalho de } \\
\text { parto. }\end{array}$ \\
\hline Kazeminia et al., 2020 & $\begin{array}{l}\text { The Effect of Lavender } \\
\text { (Lavandula stoechas L.) on } \\
\text { Reducing Labor Pain: A } \\
\text { Systematic Review and } \\
\text { Meta-Analysis }\end{array}$ & $\begin{array}{l}\text { Determinar o efeito da alfazema na dor do } \\
\text { parto no Irã usando meta-análise. }\end{array}$ & $\begin{array}{l}\text { Revisão de literatura usando os } \\
\text { artigos publicados em bancos de } \\
\text { dados nacionais e internacionais de } \\
\text { SID, MagIran, IranMedex, IranDoc, } \\
\text { Google Scholar, Biblioteca Cochrane, } \\
\text { Embase, ScienceDirect, Scopus, } \\
\text { PubMed e Web of Science (ISI). }\end{array}$ \\
\hline
\end{tabular}




\begin{tabular}{|c|c|c|c|}
\hline Horter et al., 2020 & $\begin{array}{l}\text { Dancing During Labor: Are } \\
\text { Women Down to Boogie? }\end{array}$ & $\begin{array}{l}\text { Avaliar a disposição das mulheres grávidas } \\
\text { em participar de um estudo futuro que avalie } \\
\text { a dança de baixo impacto durante o trabalho } \\
\text { de parto. }\end{array}$ & $\begin{array}{l}\text { Uma amostra de conveniência de } \\
\text { mulheres grávidas que falam inglês } \\
\text { que se apresentaram para atendimento } \\
\text { pré-natal em } 1 \text { de } 3 \text { clínicas em um } \\
\text { grande sistema de saúde integrado no } \\
\text { sudeste de Wisconsin. }\end{array}$ \\
\hline Oliveira et al., 2020 & $\begin{array}{l}\text { Uso de medidas não } \\
\text { farmacológicas para alívio } \\
\text { da dor no trabalho de parto } \\
\text { normal }\end{array}$ & $\begin{array}{l}\text { Verificar na literatura, a utilização de } \\
\text { medidas não farmacológicas para amenizar a } \\
\text { dor no trabalho de parto normal na mulher. }\end{array}$ & $\begin{array}{l}\text { Revisão de literatura usando as bases } \\
\text { de dados: SciELO, Google } \\
\text { Acadêmico e no agrupador de bases } \\
\text { de dados BVS e PUBMED. }\end{array}$ \\
\hline Biana et al., 2021 & $\begin{array}{l}\text { Non-pharmacological } \\
\text { therapies applied in } \\
\text { pregnancy and labor: an } \\
\text { integrative review }\end{array}$ & $\begin{array}{l}\text { Identificar as terapias não farmacológicas } \\
\text { aplicadas durante a gravidez e o parto. }\end{array}$ & $\begin{array}{l}\text { Revisão integrativa realizada nas } \\
\text { bases de dados: PubMed, SciELO e } \\
\text { PEDro. }\end{array}$ \\
\hline $\begin{array}{l}\text { Mascarenhas et al., } \\
2019\end{array}$ & $\begin{array}{l}\text { Evidências científicas sobre } \\
\text { métodos não } \\
\text { farmacológicos para alívio } \\
\text { da dor no parto }\end{array}$ & $\begin{array}{l}\text { Identificar na literatura nacional e } \\
\text { internacional, estudos sobre a eficácia de } \\
\text { métodos não farmacológicos na redução da } \\
\text { dor do parto. }\end{array}$ & $\begin{array}{l}\text { Revisão integrativa realizada nas } \\
\text { bases de dados MEDLINE/PUBMED, } \\
\text { SCOPUS, CINAHL, LILACS e } \\
\text { BDENF. }\end{array}$ \\
\hline $\begin{array}{l}\text { Hillmann \& Stamm, } \\
2019\end{array}$ & $\begin{array}{l}\text { Knowledge, attitude and } \\
\text { practice } \\
\text { pharmacological methods } \\
\text { of labor analgesia }\end{array}$ & $\begin{array}{l}\text { Avaliar o conhecimento, a atitude e a prática } \\
\text { de obstetras em relação aos métodos } \\
\text { farmacológicos de analgesia do parto. }\end{array}$ & $\begin{array}{l}\text { Estudo transversal (38 obstetras que } \\
\text { trabalham em maternidades públicas). } \\
\text { Foi aplicado um questionário } \\
\text { estruturado sobre conhecimentos, } \\
\text { atitudes e práticas em relação aos } \\
\text { métodos farmacológicos sistêmicos e } \\
\text { regionais. }\end{array}$ \\
\hline
\end{tabular}

Fonte: Autores.

A dor é uma realidade do momento do trabalho de parto (TP) e o determinante mais perceptível do momento do parto. Ela é decorrente da dilatação cervical, da contração uterina e da extensão do útero e alongamento da vagina. O TP é dividido em três etapas: o primeiro diz respeito ao processo de dilatação cervical completa; o segundo é o período desde a dilatação completa até o nascimento do bebê; e o terceiro é do nascimento até a dequitação da placenta (Tabatabaeichehr; Mortazavi, 2020; Raana; Fan, 2020). A dor da mulher no período do parto pode ser extremamente intensa, com tensão corporal, ansiedade e medo, o que pode piorar esse momento, por isso, o controle eficaz e satisfatório do desconforto ocasionado pela dor, deve ser individualizado para cada gestante, por meio de algumas técnicas estratégicas que podem quebrar o ciclo medo-tensão-dor (Smith et al., 2020).

Embora a dor seja um importante sinal biológico do parto, ela deve ser tratada de maneira adequada, a fim de prevenir as complicações obstétricas e, consequentemente, as intervenções médicas. Além disto, o manejo inadequado da dor no TP pode gerar consequências psicológicas negativas, colaborando para o aumento da ansiedade da parturiente, o que contribui para a elevação do estímulo do sistema nervoso simpático que, por sua vez, libera hormônios como noradrenalina, cortisol e adrenalina, que aumentam o estresse e a intensidade da dor, além da duração do TP (Mascarenhas et al., 2019; Tabatabaeichehr; Mortazavi, 2020). Nesse sentido, o medo com relação às complicações e o desejo materno são fatores importantes diante do aumento das taxas de cesárea eletiva: segundo a Organização Mundial de Saúde, a taxa ideal de cesarianas aceitáveis está entre 10\% a 15\% para se obter ótimos resultados maternos e perinatais, contudo, no Brasil, 55,5\% dos partos são realizados por cesariana (Mascarenhas et al., 2019).

Em contrapartida, quando comparado a cesariana, o parto normal é a forma natural de nascer e apresenta-se como um método mais seguro e com menor tempo de internação para mãe, todavia, a dor e a ansiedade são barreiras para a tomada de decisão de gestantes a favor do parto normal. Sendo assim, a partir da premissa que o conjunto dor-ansiedade são grandes responsáveis pelo aumento do número de cesarianas, faz-se necessário a aplicabilidade também de métodos alternativos, não farmacológicos, com o propósito de promover a maior sensação de alívio da dor e da ansiedade (Raana; Fan, 2020; Mascarenhas, 2019). 
Alguns estudos a respeito de métodos não farmacológicos demonstraram boa eficácia para redução da dor do trabalho de parto e passaram a ser uma opção para substituir a analgesia durante o TP e auxiliar a parturiente a lidar com suas queixas álgicas, são exemplos: massagem, reflexologia, acupuntura, bola suíça, técnicas mente-corpo, hipnose, aromaterapia, musicoterapia, hidroterapia, estimulação elétrica transcutânea, entre outras (Santiváñez-Acosta et al., 2020; Mascarenhas, 2019; Oliveira et al., 2020).

Segundo Santiváñes-Acosta et al., a musicoterapia pode ter impacto benéfico em reduzir a dor e a ansiedade durante o TP, principalmente em primíparas. Smith et al. também concluiu que a massagem terapêutica pode ter efeito positivo na redução da dor e na melhora da experiência emocional do parto, visto que ela, em comparação aos cuidados habituais, reduziu a ansiedade durante a primeira fase do TP (MD - 16,27; IC: 95\% - 27,3 a 5,51; 60 mulheres). A massagem ajuda a relaxar os músculos tensos do corpo, inibindo a transmissão sensorial nas vias da dor - já que a pressão advinda dela pode impedir o processamento de estímulos dolorosos, uma vez que as fibras de pressão são mais mielinizadas e transmitem sinais mais rapidamente do que as fibras de dor - ou melhorando o fluxo sanguíneo, o que faz com que muitas mulheres sintam-se reconfortadas durante o tempo de parto. Ainda segundo o mesmo estudo, a reflexologia - que consiste na aplicação de pressão digitálica nos pés - resulta em um efeito anestesiante nas demais partes do corpo; aplicar pressão nas "zonas reflexas" pode contribuir para a homeostase, reduzindo a tensão e o estresse, o que pode ser útil para diminuir o desconforto pré e pós-natal.

Nesse mesmo sentido, Tabatabaeichehr e Mortazavi verificaram 33 estudos e a maioria atestou a eficácia da aromaterapia na redução do estresse e da algia durante o parto. Uma outra revisão sistemática e de meta-análise publicada por Kazeminia et al., demonstrou que a inalação do aroma da lavanda e alfazema reduziu os níveis de ansiedade e a secreção de cortisol e aumentou a secreção de serotonina no trato gastrointestinal. Em estudos com animais, a essência oleosa da lavanda revelou ter efeito sedativo e analgésico, já que as cetonas da planta reduzem a dor, a inflamação e ajudam a adormecer, o que demonstra o poder tranquilizador da mesma.

Em contrapartida, sobre a acupuntura, o estudo de Smith et al. revelou que, entre acupuntura e acupressão, não houve conclusão sobre um ser mais benéfico em relação ao outro, com relação a diminuição da dor. Demonstrou-se que a acupuntura pode vir a aliviar a dor e reduzir o uso de analgésicos farmacológicos, entretanto, são necessárias mais pesquisas devido às baixas evidências até o momento.

A motivação para a prática dos métodos não farmacológicos foi a diminuição da dor. Em alguns estudos foram relatados que a dor pode ser minimizada com uso dos métodos. A deambulação nos momentos iniciais do trabalho de parto é uma forma de tentar amenizar a dor, que ainda não é tão presente. É um método confortável visto que a gravidade pode estar ao seu favor promovendo a rotação adequada do feto na pelve. O bem estar e a satisfação emocional da parturiente passam pelo alivio da dor como um dos recursos para o cuidado e conforto, e a experiência com a dor será de maneira mais tranquila. A atenção voltada para atender as necessidades especificas do alivio da dor pode ajudar a ter um trabalho de parto mais satisfatório (Mielke et al., 2019).

\section{Conclusão}

O parto pode ser a o momento mais importante da vida de uma mulher e, também, muitas vezes, o instante mais doloroso de sua vida. Por isso, é muito relevante que as gestantes tenham neste período todo o conforto preciso e que não necessariamente sejam submetidas à rotina medicamentosa, como ocorre em muitas ocasiões.

Já é conhecido que existem outros métodos não farmacológicos que atuam efetivamente na diminuição da dor e da ansiedade durante o parto - banho morno, exercícios respiratórios, massagem, bola suíça, aromaterapia, musicoterapia, entre 
outros - e tais medidas devem ser disseminadas a fim de propiciar uma melhor experiência e conforto intra-parto para as gestantes.

Para estudos futuros, sugere-se mais pesquisas sobre a eficácia de todos os métodos não farmacológicos utilizados até hoje, para que, assim, com mais volume de pesquisas, confirme-se a tese de que essas técnicas são realmente eficazes para redução da dor e da ansiedade decorrentes do período de parto e possam, dessa forma, serem mais implementadas em serviços obstétricos.

\section{Referências}

Almeida, N. A. M., Soares, L. J., Sodré, R. L. R., \& Medeiros, M. (2008). A dor do parto na literatura científica da Enfermagem e áreas correlatas indexada entre 1980-2007. Revista Eletrônica de Enfermagem, 10(4). https://doi.org/10.5216/ree.v10.46818

Biana, C. B., Cecagno, D., Porto, A. R., Cecagno, S., Marques, V. de A., \& Soares, M. C. (2021). Non-pharmacological therapies applied in pregnancy and labor: an integrative review. Revista Da Escola de Enfermagem Da USP, 55. https://doi.org/10.1590/s1980-220x2019019703681

Da, C., Alves, C., Bispo Cavalcante, M., Claudia Costa De Sampaio, A., Linhares, H., Oliveira, E., \& Teixeira, M. (n.d.). Humanização do parto a partir de métodos não farmacológicos para o alívio da dor: relato de experiência. https://sanare.emnuvens.com.br/sanare/article/viewFile/870/530

Gayeski, M. E., \& Brüggemann, O. M. (2010). Métodos não farmacológicos para alívio da dor no trabalho de parto: uma revisão sistemática. Texto \&Contexto - Enfermagem, 19(4), 774-782. https://doi.org/10.1590/s0104-07072010000400022

Getu, A. A., Getie, S. A., Gela, G. B., Maseresha, E. A., Feleke, B. E., \& Muna, A. M. (2020). Non-pharmacological labor pain management and associated factor among skilled birth attendants in Amhara Regional State health institutions, Northwest Ethiopia. Reproductive Health, 17(1). https://doi.org/10.1186/s12978-020-01043-1

Hillmann, B. R., \& Stamm, A. M. N. de F. (2019). Knowledge, attitude and practice regarding pharmacological methods of labor analgesia. Brazilian Journal of Pain, 2(1). https://doi.org/10.5935/2595-0118.20190004

Horter, D. A., Heslin, K., Forgie, M., Malloy, E., \& Kram, J. J. F. (2020). Dancing During Labor: Are Women Down to Boogie? Journal of Patient-Centered Research and Reviews, 7(4), 349-354. https://doi.org/10.17294/2330-0698.1746

Kazeminia, M., Abdi, A., Vaisi-Raygani, A., Jalali, R., Shohaimi, S., Daneshkhah, A., Salari, N., \& Mohammadi, M. (2020). The Effect of Lavender (Lavandula stoechas L.) on Reducing Labor Pain: A Systematic Review and Meta-Analysis. Evidence-Based Complementary and Alternative Medicine, 2020, 1-11. https://doi.org/10.1155/2020/4384350

Leal, M. do C., Pereira, A. P. E., Domingues, R. M. S. M., Filha, M. M. T., Dias, M. A. B., Nakamura-Pereira, M., Bastos, M. H., \& Gama, S. G. N. da. (2014). Intervenções obstétricas durante o trabalho de parto e parto em mulheres brasileiras de risco habitual. Cadernos de Saúde Pública, 30(suppl 1), S17S32. https://doi.org/10.1590/0102-311x00151513

Mahbubeh Tabatabaeichehr, \& Hamed Mortazavi. (2020). The Effectiveness of Aromatherapy in the Management of Labor Pain and Anxiety: A Systematic Review. Ethiopian Journal of Health Sciences, 30(3). https://doi.org/10.4314/ejhs.v30i3.16

Mascarenhas, V. H. A., Lima, T. R., Silva, F. M. D. e, Negreiros, F. dos S., Santos, J. D. M., Moura, M. Á. P., Gouveia, M. T. de O., \& Jorge, H. M. F. (2019). Evidências científicas sobre métodos não farmacológicos para alívio a dor do parto. Acta Paulista de Enfermagem, $32(3)$, 350-357. https://doi.org/10.1590/1982-0194201900048

Mielke, K. C., Gouveia, H. G., \& Gonçalves, A. D. C. (2019). A prática de métodos não farmacológicos para o alívio da dor de parto em um hospital universitário no Brasil. Avances En Enfermería, 37(1), 47-55. https://doi.org/10.15446/av.enferm.v37n1.72045

Oliveira, L. S., Oliveira, L. K. P., Rezende, N. C. C. G., Pereira, T. L., \& Abed, R. A. (2020). Uso de medidas não farmacológicas para alívio da dor no trabalho de parto normal. Brazilian Journal of Health Review, 3(2), 2850-2869. https://doi.org/10.34119/bjhrv3n2-128

Osório, S. M. B., Silva Júnior, L. G. da, \& Nicolau, A. I. O. (2014). Assessment of the effectiveness of non-pharmacological methods in pain relief during labor. Revista Da Rede de Enfermagem Do Nordeste, 15(1). https://doi.org/10.15253/2175-6783.2014000100022

Raana, H. N., \& Fan, X.-N. (2020). The effect of acupressure on pain reduction during first stage of labour: A systematic review and metaanalysis. Complementary Therapies in Clinical Practice, 39, 101126. https://doi.org/10.1016/j.ctcp.2020.101126

Santiváñez-Acosta, R., Tapia-López, E. de las N., \& Santero, M. (2020). Music Therapy in Pain and Anxiety Management during Labor: A Systematic Review and Meta-Analysis. Medicina, 56(10), 526. https://doi.org/10.3390/medicina56100526

Santos, A. C. de M., Nascimento, C. D. do, Campos, T. C. de, \& Sousa, N. N. A. G. de. (2021). Atuação Da Enfermagem No Uso De Métodos Não Farmacológicos Para O Alívio Da Dor Durante O Trabalho De Parto/ Ursing Performance In The Use Of Non-Pharmacological Methods For Pain Relief During Child Labor. Brazilian Journal of Development, 7(1), 9505-9515. https://doi.org/10.34117/bjdv7n1-643

Smith, C. A., Collins, C. T., Levett, K. M., Armour, M., Dahlen, H. G., Tan, A. L., \& Mesgarpour, B. (2020). Acupuncture or acupressure for pain management during labour. Cochrane Database of Systematic Reviews. https://doi.org/10.1002/14651858.cd009232.pub2 
Research, Society and Development, v. 10, n. 11, e156101119443, 2021

(CC BY 4.0) | ISSN 2525-3409 | DOI: http://dx.doi.org/10.33448/rsd-v10i11.19443

Smith, C. A., Levett, K. M., Collins, C. T., \& Jones, L. (2012). Massage, reflexology and other manual methods for pain management in labour. Cochrane Database of Systematic Reviews. https://doi.org/10.1002/14651858.cd009290.pub2

Smith, C. A., Levett, K. M., Collins, C. T., Armour, M., Dahlen, H. G., \& Suganuma, M. (2018). Relaxation techniques for pain management in labour. Cochrane Database of Systematic Reviews. https://doi.org/10.1002/14651858.cd009514.pub2 